\title{
Resposta hipertensiva ao esforço: um problema silencioso
}

Brenda Domingues, * Vanessa Xavier,* Teresa Marcos**

\section{RESUMO}

Introdução: As pessoas fisicamente ativas são habitualmente consideradas saudáveis e protegidas de doença cardiovascular e de hipertensão pela manutenção de uma boa condição física. De facto, a prevalência de hipertensão nestes grupos calcula-se ser cerca de $50 \%$ inferior em relação à da população geral, sendo o exercício físico uma medida não farmacológica para controlo do risco cardiovascular.

É objetivo deste trabalho sensibilizar os profissionais de saúde para o problema da hipertensão arterial de esforço (hipertensão incidental) e para a necessidade de um diagnóstico precoce de maneira a rastrear e controlar o risco cardiovascular destes doentes. Descrição de caso: JFR, sexo masculino, 33 anos, caucasiano, assintomático. Ex-atleta de alta competição (praticante de natação pura durante 12 anos, entre os 10 e os 22 anos de idade); atualmente praticante regular de exercício físico de moderada/alta intensidade. Fumador (cinco UMA), com um IMC de $28,9 \mathrm{~kg} / \mathrm{m}^{2}$ e com antecedentes familiares de obesidade e hipertensão arterial. No contexto de exames de avaliação clínica de "rotina" detetaram-se valores tensionais borderline (normal/alto), excesso ponderal, hipercolesterolemia (com um índice colesterol total/colesterol HDL de 4,7), hipertrofia ventricular esquerda por critérios eletro e ecocardiográficos; a monitorização ambulatória da pressão arterial de $24 \mathrm{~h}$ não evidenciou hipertensão arterial. No seguimento da avaliação médica, verificou-se uma prova de esforço com franca resposta hipertensiva, o que permitiu não só explicar as anomalias cardíacas encontradas, mas também intervir com medidas farmacológicas precoces que se mostraram eficazes no controlo da mesma. Comentário: Em pessoas assintomáticas e normotensas, uma resposta tensional exagerada durante o exercício está associada a um risco aumentado de desenvolvimento de hipertensão, com consequente risco de repercussões cardiovasculares negativas. A prova de esforço pode ser útil para identificar indivíduos com respostas anormais, eventualmente percussoras de hipertensão, permitindo uma intervenção precoce na história natural da doença. Este caso salienta a importância de se conhecerem os estilos de vida dos doentes para que a avaliação clínica seja o mais correta possível.

Palavras-chave: Hipertensão; Teste de Esforço; Pré-hipertensão.

\section{INTRODUÇÃO}

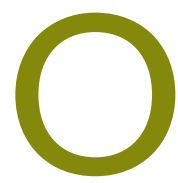

exercício é uma medida conhecida de prevenção primária de hipertensão arterial (HTA). ${ }^{1}$ Na realidade, os atletas e os indivíduos fisicamente ativos são habitualmente considerados protegidos de doença cardiovascular e de hipertensão pela manutenção de uma boa condição física. ${ }^{2}$ De facto, a prevalência de hipertensão calcula-se ser cerca de $50 \%$ inferior à da população geral nestes dois grupos de indivíduos. No entanto, o risco da doença está au-

* Médica Interna de Medicina Geral e Familiar. ACeS Porto Oriental - UCSP Vale Formoso, Porto, Portugal.

**Médica Especialista de Medicina Geral e Familiar. ACeS Porto Oriental - UCSP Vale Formoso, Porto, Portugal. mentado na raça negra, nos idosos, obesos e indivíduos com história familiar de hipertensão. ${ }^{1-2}$

Em pessoas assintomáticas e normotensas, uma resposta tensional exagerada com o esforço associa-se a um risco aumentado de HTA futura, bem como a repercussões negativas a nível cardiovascular. ${ }^{1-15}$

É objetivo deste trabalho a sensibilização dos profissionais de saúde para o problema da hipertensão arterial de esforço e respetiva necessidade de deteção precoce, de maneira a rastrear e controlar o risco cardiovascular destes doentes.

\section{DESCRIÇÃO DE CASO}

Caracterização do utente: JFR, género masculino, 
caucasiano, 33 anos de idade, gestor, casado e com um filho (família nuclear na fase II do ciclo de Duvall), natural e residente no Porto.

Apresenta antecedentes pessoais de duas fraturas desportivas (escafoide esquerdo e $3 .^{\circ}$ metacarpo esquerdo) e é ex-atleta de alta competição (praticante de natação pura durante 12 anos, entre os 10 e os 22 anos de idade). Desconhece alergias e nega medicação habitual. É fumador desde os 16 anos (cinco UMA) e refere um consumo esporádico de álcool. Tem uma alimentação equilibrada, sem restrições. Pratica atualmente (e desde há 10 anos) exercício físico regular de intensidade moderada a alta (futebol, corrida, bicicleta e natação). A história familiar revelou mãe de 62 anos com antecedentes de hipertensão arterial, excesso de peso, psoríase e osteoporose e pai de 61 anos com obesidade central, neoplasia maligna do cólon aos 51 anos e sarcoma de kaposiendémico aos 60 anos (Figura 1).

Caso: A 15 de fevereiro de 2010, JFR, à data com 33 anos, recorreu ao médico assistente solicitando "análises de rotina", apesar de assintomático. A sua última avaliação médica tinha sido aos 26 anos. Ao exame objetivo apresentava uma pressão arterial normal-alta $(139 / 81 \mathrm{mmHg})$ e excesso de peso (peso de $100 \mathrm{~kg}$, IMC de $28,9 \mathrm{~kg} / \mathrm{m}^{2}$ ). A restante avaliação física não apresentava alterações, nomeadamente a auscultação cardíaca e pulmonar revelaram-se normais, pulsos arteriais sem alterações, palpáveis e simétricos e não apresentava sopros abdominais ou outros. Foram identificados, como problemas, o abuso do tabaco, o excesso de peso e a pressão arterial (PA) elevada, sendo solicitados exames auxiliares de diagnóstico: hemograma, glicose, creatinina, urina tipo II, colesterol total, colesterol HDL, triglicerídeos e eletrocardiograma. Foi solicitada automonitorização da pressão arterial em ambulatório uma vez por semana durante oito semanas e aconselhadas medidas higiénico-dietéticas, com vista à perda ponderal, bem como diminuição de ingestão salina e de gorduras saturadas. Foi realizada uma intervenção

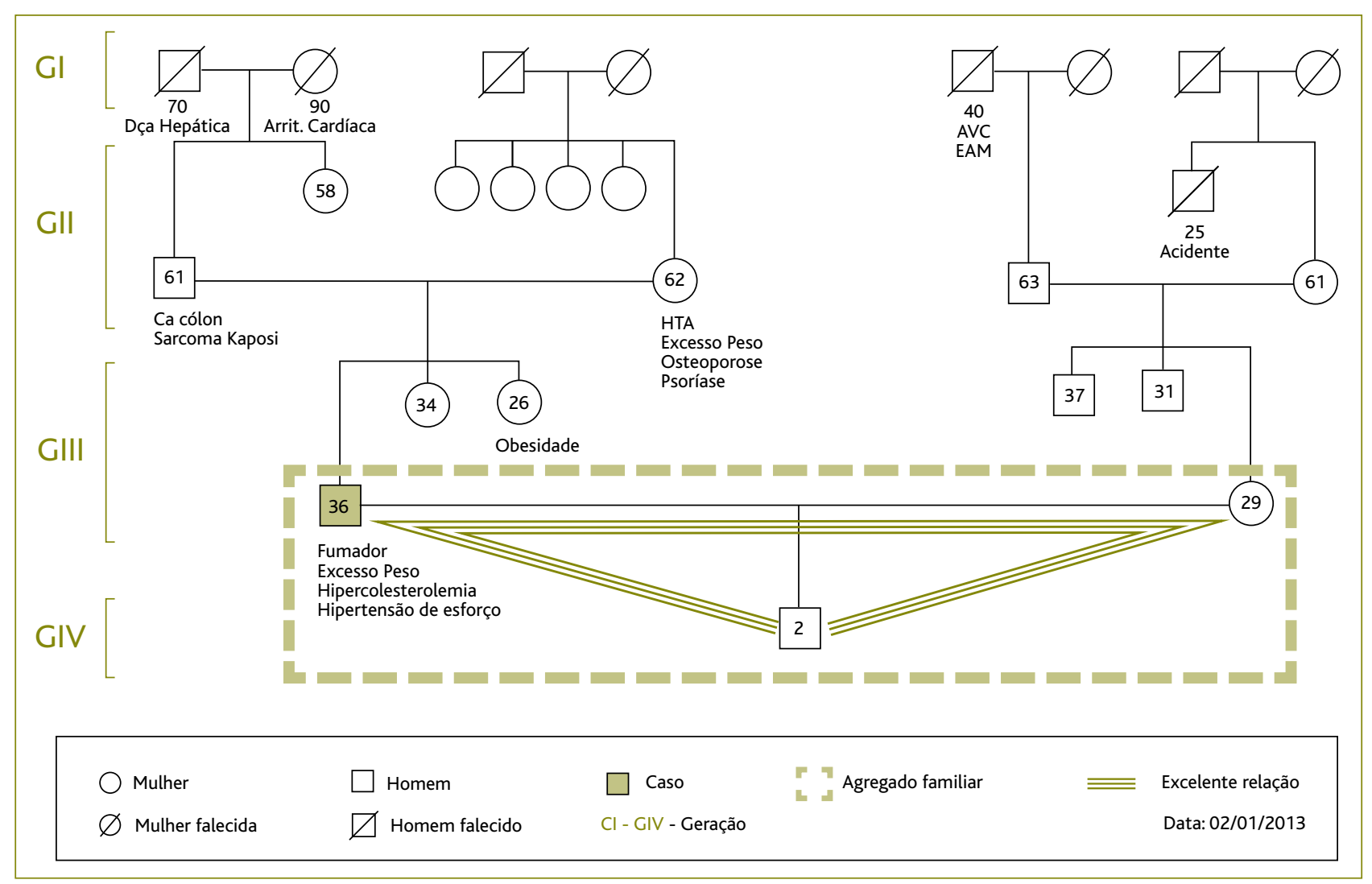

Figura 1. Genograma. 
breve em relação aos hábitos tabágicos, não estando o doente motivado para deixar de fumar.

Passados cerca de dois meses recorreu a consulta de cardiologia particular por alterações no eletrocardiograma (ECG) (bradicardia sinusal - 50bpm; hipertrofia ventricular esquerda por critérios de voltagem e alterações da repolarização ventricular compatíveis com sobrecarga e/ou isquemia) (Figura 2). Realizou ecocardiograma que mostrou "dilatação ligeira da aurícula esquerda $(44 \mathrm{~mm})$ ” (sem referência no relatório a correção à superfície corporal), "ligeira hipertrofia septal (13mm), (...)

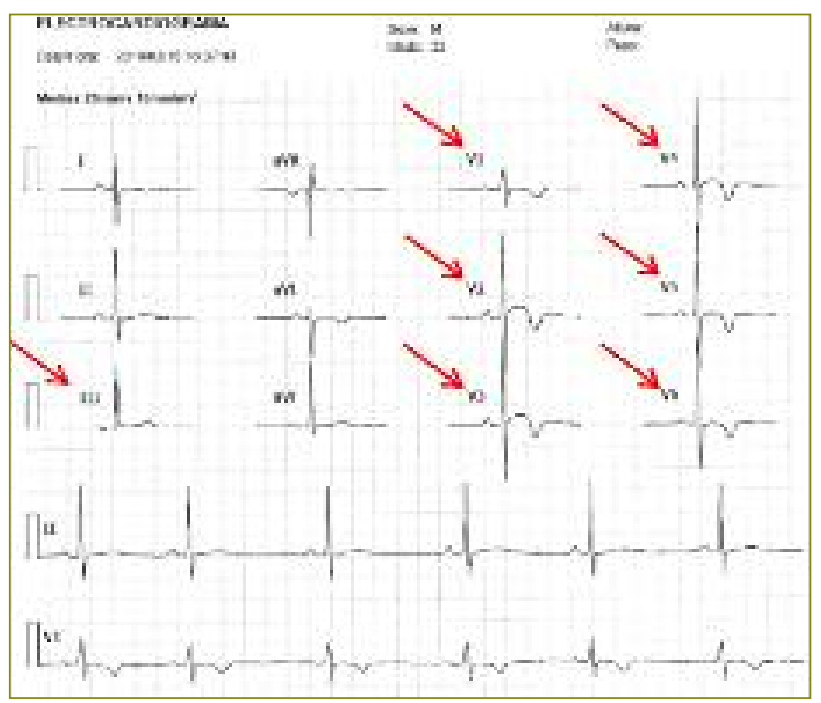

Figura 2. ECG inicial (15.03.2010).

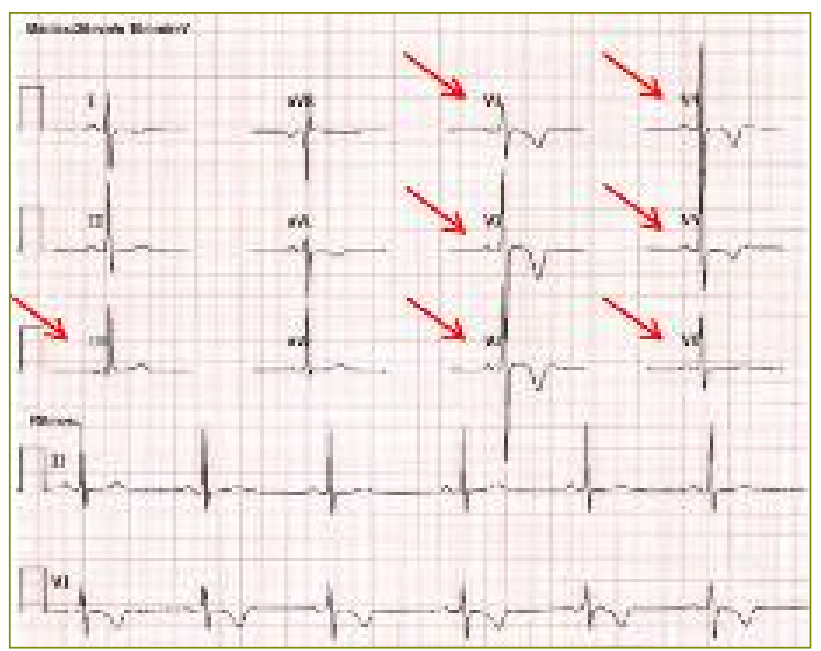

Figura 3. ECG de reavaliação (26.09.2011). com espessura normal das restantes paredes ventriculares; ausência de sinais indiretos de hipertensão pulmonar e boa função bi-ventricular sistólica" (a fração de encurtamento não é mencionada no relatório). As alterações foram interpretadas pelo médico cardiologista como "coração de atleta”.

Só mais de um ano depois (05.05.2011 - 34 anos) voltou novamente à consulta de MGF com os mesmos exames auxiliares de diagnóstico. Manteve-se assintomático desde a última consulta e a avaliação clínica revelou perda ponderal ligeira, sem outras alterações (peso de 94,8kg, IMC de 27,4kg/m², PA 140/80mmHg). Analiticamente foi diagnosticada hipercolesterolemia (colesterol total $261 \mathrm{mg} / \mathrm{dL}$, colesterol HDL 55mg/dL e colesterol LDL 176mg/dL, com índice CT/HDL de 4,7) e a automonitorização da pressão arterial em ambulatório não mostrou alterações (média < 135/85mmHg).

Considerando as alterações dos exames auxiliares de diagnóstico e a idade do doente, optou-se por repetir o ECG e ecocardiograma para reavaliação da evolução, após um ano, da hipertrofia septal anteriormente detetada e solicitar a monitorização ambulatória da pressão arterial (MAPA) de 24h. Foi ainda reforçado o aconselhamento dietético, nomeadamente em relação à redução do consumo de gorduras saturadas e carnes vermelhas, mantendo-se sem terapêutica hipolipemiante dado o baixo risco cardiovascular.

Após seis meses mantinha o mesmo IMC e valores sobreponíveis de pressão arterial (peso de 93kg, IMC $26,9 \mathrm{~kg} / \mathrm{m}^{2}$, PA 132/80mmHg). O ECG e ecocardiograma mantinham as alterações prévias (Figura 3 e 4). A MAPA de $24 \mathrm{~h}$ (21.11.2011) não revelou alterações relevantes, não havendo critérios de hipertensão arterial.

Considerando a persistência das alterações, não se tendo detetado motivo para as mesmas, a idade do doente, assim como a existência de fatores de risco cardiovascular (hipercolesterolemia, excesso de peso, hábitos tabágicos e história familiar de hipertensão arterial) e a prática desportiva moderada a intensa, foi solicitada uma prova de esforço (28.11.2011) que revelou “... resposta hipertensiva ao esforço com necessidade de interrupção da mesma por PAS $>250 \mathrm{mmHg}$ (PA 252/89mmHg ao $8^{\circ}$ minuto - estadio 2 do protocolo de Bruce). Prova negativa para isquemia do miocárdio". Neste contexto foi decidido iniciar terapêutica farmacológica com Ramipril 5mg/dia. Posteriormente, foi feita reavaliação sob terapêutica anti- 


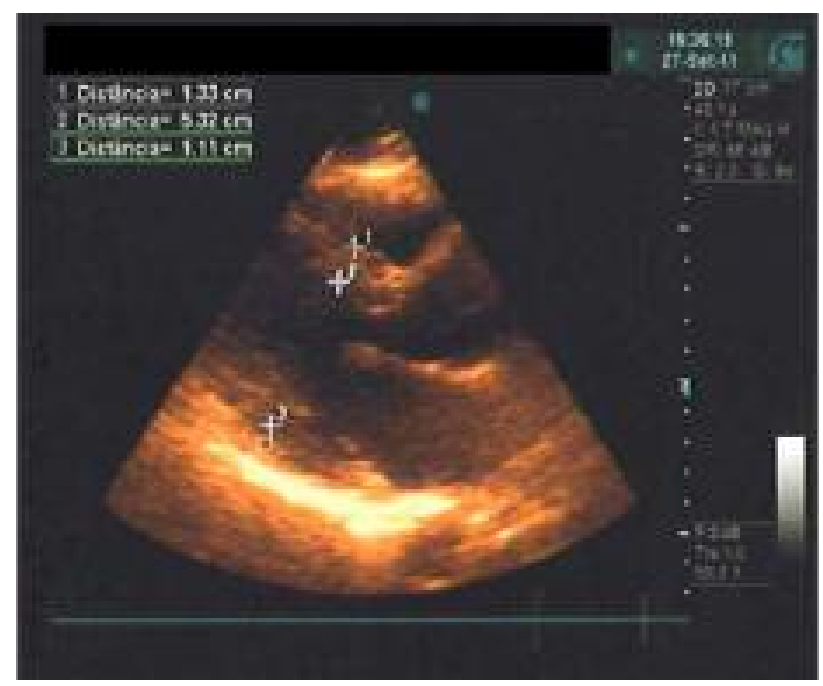

Figura 4. Ecocardiograma (27.09.2011).

-hipertensora com PA máxima de 176/70mmHg no pico do esforço: "prova de esforço máxima interrompida por fadiga muscular aos 12:30 minutos (estadio 4 do protocolo de Bruce). Frequência cardíaca máxima atingida de 161 bpm (87\% da FC teórica máxima prevista). Não referiu sintomas. Capacidade de esforço normal (12.6 METS - 100\% capacidade teórica prevista). Resposta tensional ao esforço normal. Pseudo-normalização do segmento ST e da onda T. Prova de esforço normal" (Figura 5).

\section{COMENTÁRIO}

Este caso salienta a importância da suspeição clínica num contexto menos frequente, onde os resultados dos exames complementares de diagnóstico apresentam alterações não enquadráveis num indivíduo aparentemente saudável.

A hipertensão arterial começa frequentemente na idade adulta, aumentando a sua prevalência com o avançar da idade. ${ }^{10} \mathrm{~A}$ prova de esforço, tradicionalmente utilizada para o diagnóstico de doença coronária, não se esgota no rastreio ou follow-up da cardiopatia isquémica, tendo outras indicações, como a avaliação do perfil tensional ou a avaliação do comportamento de arritmias, sendo estas indicações muitas vezes esquecidas na rotina do dia-a-dia; é neste contexto que se justifica a avaliação da resposta cardiovascular ao exercício, em particular nos desportistas com 35 ou mais anos de idade..$^{11}$ É um teste essencial para identificar indivíduos com respostas anormais, nomeadamente respostas hipertensivas ao esfor-

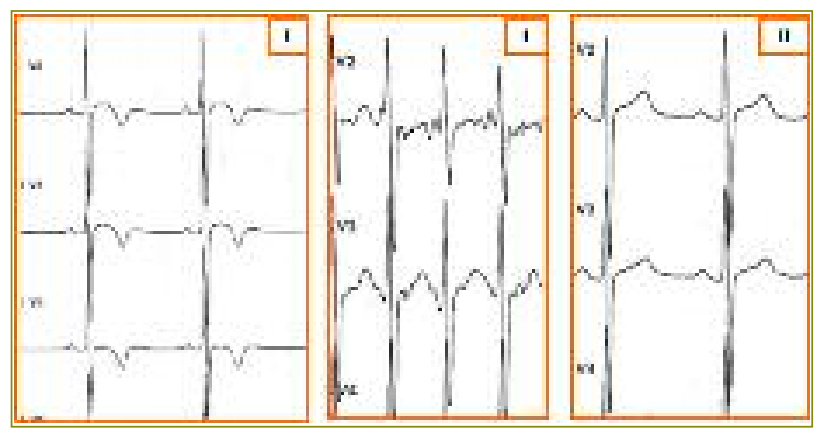

Figura 5. Prova de esforço (17.01.2013) Pseudo-normalização do segmento ST. I - Repouso; II - Pico de esforço; III - Recuperação.

ço, eventualmente percursoras de hipertensão arterial, ${ }^{12}$ permitindo modificar a estratificação do risco cardiovascular (CV) e diminuindo a probabilidade de morte súbita/eventos cardiovasculares.

A prova de esforço é um exame não invasivo, seguro, pouco dispendioso, de fácil acesso e fácil execução, sendo essencial para identificar indivíduos com respostas anormais e, portanto, um teste de estratificação de risco e predição de futuros eventos CV. Vários estudos demonstraram que o aumento excessivo dos valores da pressão arterial, em resposta ao exercício, pode ser um fator preditivo do desenvolvimento futuro de HTA com consequente aumento do risco CV. ${ }^{1-16}$

Esta resposta hipertensiva ao esforço parece ser explicada por uma falência nos mecanismos fisiológicos de adaptação ao esforço, nomeadamente por alterações da vasoregulação com incapacidade de reduzir a resistência vascular periférica como resultado de alterações vasculares iniciais que antecedem o estabelecimento da HTA.,12,14-16

A identificação deste problema permite, assim, uma intervenção precoce, preventiva e eficaz na história natural da doença, sendo recomendada, como primeira opção terapêutica, o uso de um inibidor da enzima de conversão da angiotensina ou um antagonista dos canais de cálcio não dihidropiridínico.--2,16

\section{REFERÊNCIAS BIBLIOGRÁFICAS}

1. Pescatello LS, Franklin BA, Fagard R, Farquhr WB, Kelley GA, Ray CA, et al. American College of Sports Medicine position stand: exercise and hypertension. Med Sci Sports Exerc. 2004;36(3):533-53.

2. Niedfeldt MW. Managing hypertension in athletes and physically active patients. Am Fam Physician. 2002;66(3):445-52.

3. Fletcher GF, MillsWC, TaylorWC. Update on exercise stress testing. Am Fam Physician. 2006;74(10):1749-54. 
4. Miyai N, Arita M, Morioka I, Miyashita K, Nishio I, Takeda S. Exercise BP response in subjects with high-normal BP: exaggerated blood pressure response to exercise and risk of future hypertension in subjects with high-normal blood pressure. J Am Col Cardiol. 2000;36(5):1626-31.

5. Holmqvist L, Mortensen L, Kanckos C, Ljungman C, Mehlig K, Manhem $K$. Exercise blood pressure and the risk of future hypertension. J Hum Hypertens. 2012;26(12):691-5.

6. Sharma K, Kohli P, Gulati M. An update on exercise stress testing. Curr Probl Cardiol. 2012;37(5):177-202.

7. Matthews CE, Pate RR, Jackson KL, Ward DS, Macera CA, Kohl HW, et al. Exaggerated blood pressure response to dynamic exercise and risk of future hypertension. J Clin Epidemiol. 1998;51(1):29-35.

8. Farah R, Shurtz-Swirski R, Nicola M. High blood pressure response to stress ergometry could predict future hypertension. Eur J Intern Med. 2009;20(4):366-8.

9. Lima SG, Albuquerque MF, Oliveira JR, Ayres CF, Cunha JE, Oliveira DF, et al. Exaggerated blood pressure response during the exercise treadmill test as a risk factor for hypertension. Braz J Med Biol Res. 2013;46(4):368-47.

10. De Macedo ME, Lima MJ, Silva AO, Alcântara P, Ramalhinho V, Carmona J. Prevalence, awareness, treatment and control of hypertension in Portugal: the PAP study. Rev Port Cardiol. 2007;26(1):21-39.

11. Thompson PD. Preparticipation health screening. In Pescatello LS, editor.ACSM's guidelines for exercise testing and prescription. 9th ed. Philadelphia: Lippincott Williams \& Wilkins; 2013. p. 19-38. ISBN 9781609136055
12. Singh JP, Larson MG, Manolio TA, O'Donnell CJ, Lauer M, Evans JC, et al. Blood pressure response during treadmill testing as a risk factor for new-onset hypertension: the Framingham heart study. Circulation. 1999;99(14):1831-6.

13. Weiss SA, Blumenthal RS, Sharrett AR, Redberg RF, Mora S. Exercise blood pressure and future cardiovascular death in asymptomatic individuals. Circulation. 2010;121(19):2109-16.

14. Lima EG, Spritzer N, Herkenhoff FL, Bermudes A, Vasquez EC. Noninvasive ambulatory 24 -hour blood pressure in patients with high normal blood pressure and exaggerated systolic pressure response to exercise. Hypertension. 1995;26(6 Pt 2):1121-4.

15. Shim CY, Ha JW, Park S, Choi EY, Choi D, Rim SJ, et al. Exaggerated blood pressure response to exercise is associated with augmented rise of angiotensin II during exercise. J Am Coll Cardiol. 2008;52(4):287-92.

16. Ruivo JA, Alcântara P. Hypertension and exercise. Rev Port Cardiol. 2012;31(2):151-8.

\section{CONFLITO DE INTERESSES}

As autoras declaram não ter conflitos de interesses.

\section{ENDEREÇO PARA CORRESPONDÊNCIA}

Brenda dos Santos Domingues

Rua Padre Luís Cabral, 1079, Hb 3.2, 4150-465 Porto

E-mail: brendadomi@gmail.com

Recebido em 24-09-2014

Aceite para publicação em 16-04-2015

\section{ABSTRACT}

\section{HYPERTENSIVE RESPONSE TO EXERCISE: A SILENT PROBLEM}

Introduction: Physically active people are usually considered healthy and protected from cardiovascular disease and hypertension due to their good physical condition. The prevalence of hypertension in these groups is estimated to be about $50 \%$ lower than in the general population. Exercise is a well-known non-pharmacological measure for diminishing cardiovascular risk. The purpose of this article is to make health professionals aware of the hypertensive response to exercise and of the need for early diagnosis in order to assess and control cardiovascular risk in these patients.

Description of Case: The case of a 33 year-old asymptomatic Caucasian male is presented. He was a former high-level competitive swimmer between the ages of 10 and 22 . He currently performs regular physical activity with moderate to high intensity. His personal history includes current smoking, a BMI of $28.9 \mathrm{~kg} / \mathrm{m}^{2}$ and a family history of obesity and high blood pressure. As part of his routine assessment, we detected borderline elevated blood pressure values (high normal), overweight, hypercholesterolemia (with a total cholesterol/HDL cholesterol index of 4.7), and left ventricular hypertrophy by electrocardiographic and echocardiographic criteria. The 24-hour ambulatory blood pressure measurement was negative for hypertension. A stress test was performed. It was interrupted prematurely due to a hypertensive response to exercise. This explained not only the cardiac anomalies found but also allowed early pharmacological intervention with control of blood pressure during exercise.

Comments: In asymptomatic and normotensive individuals, an exaggerated blood pressure response during exercise is associated with an increased risk of future hypertension and negative cardiovascular outcomes. The treadmill test can be used to identify individuals with abnormal responses, probably preceding hypertension, allowing early intervention in the natural history of the disease. This case emphasizes the importance of knowing the patient's lifestyle for optimal clinical evaluation.

Keywords: Hypertension; Exercise Rest; Prehypertension. 